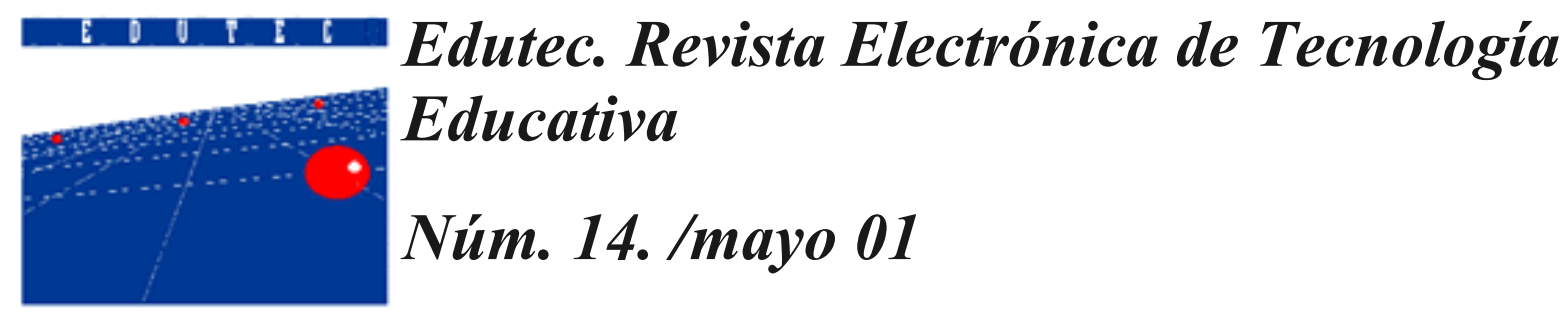

\title{
UNIVERSIDADES VIRTUALES: \\ EL CASO DE LA UNIVERSIDAD OBERTA DE CATALUNYA. (UOC)
}

\author{
Anna Pagés Santacana \\ apages@campus.uoc.es
}

Este trabajo forma parte de las actas del Seminario de Educación a Distancia para América Latina y El Caribe. Organizado por el Instituto Latinoamericano de Comunicación Educativa. Buenos Aires, 1999.

http://www.ilce.edu.mx/icde ilce/ponencia/argentina/ponencia/pages4.pdf

Podríamos afirmar que la Universitat Oberta de Catalunya es una universidad virtual porque dispone de un Campus Virtual como un espacio especialmente diseñado para agilizar al máximo el proceso de formación del estudiante. Sin embargo, el conjunto del modelo pedagógico de la Universitat Oberta de Catalunya tiene un carácter diversificado, ya que junto al Campus Virtual -el espacio de formación por excelencia- existen los centros de apoyo, los encuentros presenciales, y los materiales didácticos.

Describiremos brevemente los elementos básicos del diseño pedagógico del Campus Virtual, centrándonos en los dos ejes que lo configuran: por una parte, el equilibrio entre la individualización y la interacción; por otra parte, la reformulación de la función docente.

\section{I- El modelo universitario de la UOC}

Los sistemas educativos se enfrentan hoy a una situación de cambio, la transición de la sociedad industrial a la sociedad de la información (Tiffin \& Rajasingham, 1995), caracterizada por el desarrollo tecnológico y por nuevas formas, más flexibles y diversificadas, de organización social. Ello obliga a los responsables de la acción educativa a reflexionar sobre las dudas y las paradojas que se generan en un momento tan significativo como el actual. En el campo de la formación universitaria, los interrogantes se entrecruzan con una tradición histórica, especialmente en Europa, de mucho peso, que presenta grandes resistencias al cambio y a la adaptación. ¿Es posible pensar los procesos de formación en la universidad con los parámetros de un modelo que termina? ¿O deberíamos, quizás, situar la pedagogía y la formación en otro contexto, y desde ese lugar intentar dar una respuesta distinta a los nuevos retos y desafíos educativos de la era postindustrial?

El proyecto de formación de la Universitat Oberta de Catalunya pretende anticipar una posible respuesta a algunos de estos interrogantes, definiéndose como una institución universitaria de enseñanza no presencial que aprovecha las posibilidades que las nuevas tecnologías ofrecen, tanto en lo que se refiere a la vehiculación de contenidos como a la relación estudiante-profesor y a la de los estudiantes entre sí. El uso de las nuevas tecnologías configura el modelo didáctico de la UOC, así como su interés en la investigación sobre su aplicación didáctica. La red informática y el Campus Virtual constituyen el espacio de intercambio y de aprendizaje por excelencia, desde el cual y a través del cual se desarrolla la docencia, se promueve el deseo de saber y de formación 
del estudiante y se le hace partícipe de la institución.

Podríamos definir brevemente el modelo pedagógico de la UOC desde cuatro ejes básicos, que giran alrededor del núcleo fundamental, el estudiante:

1- Los materiales didácticos. Constituyen el instrumento para el estudio, donde se reflejan los contenidos y se distribuyen didácticamente de forma progresiva y ordenada, secuenciados, con propuestas de actividades y evaluación, para uso del estudiante. Todos los materiales editados en papel llevan también un material multimedia asociado (disquetes, vídeos...).

2- Los encuentros presenciales y los centros de apoyo al estudio, espacios para el contacto personal entre profesores y estudiantes y entre estudiantes entre sí para facilitar la creación de vínculos académicos, así como también favorecer los lazos de convivencia entre los miembros de la universidad.

3- El Campus Virtual. Cualquier estudiante que tenga un ordenador y un módem puede acceder al Campus Virtual, configurado desde una red telefónica conmutada -más adelante red digital de servicios integrados- que cubre todo el territorio de Catalunya. En síntesis, el Campus Virtual permite:

- La comunicación interactiva entre estudiantes y profesores, para todas las tareas relacionadas con la docencia y el aprendizaje: aclaración de dudas, entrega y corrección de ejercicios, debates, etc...

- La comunicación y relación entre estudiantes, que pueden crear espacios para informaciones generales ("petits anuncis"), para la discusión ("forum") o para la conversación ("bar virtual"); y profesores ("sala de consultoría/ sala de tutoría").

- El acceso a los servicios propios de la Universidad: biblioteca (consulta, préstamo de libros), secretaría (matriculaciones, gestiones de tipo administrativo), etc...

- El acceso a bases de datos internacionales como Internet.

3- El papel de los tutores y consultores, profesionales que asumen tareas distintas que perfilaremos más adelante y que ofrecen una atención y seguimiento personalizado a cada estudiante.

4- La tipología de los estudiantes. El rasgo más característico del estudiante de la UOC es su diversidad: de edades, de tiempo disponible, de antecedentes académicos y también de procedencia geográfica, lo cual requiere un sistema de gran flexibilidad didáctica que permita una adaptación individualizada de los estudios.

\section{II- Tutoría y consultoría, funciones potenciadoras del aprendizaje}

La UOC distingue dos figuras básicas en la actividad académica. El profesor-consultor, por una parte, responsable de la docencia y vinculado a los contenidos de enseñanza de una materia concreta; y el profesor-tutor, de otra, que cumple una función de motivación, seguimiento y asesoramiento de la trayectoria universitaria del estudiante.

La función fundamental del tutor es "hacer un seguimiento de los estudios, dar orientaciones generales y aconsejar de forma individualizada, en todo lo que ser refiera al progreso en los estudios en la UOC a lo largo de toda la carrera"(Guía del tutor, UOC).

En la enseñanza a distancia, a diferencia de la presencial, es fundamental evitar la sensación de aislamiento del estudiante, así como velar para que no se produzca el desánimo y el abandono de los estudios. El tutor se ocupa de que el estudiante se sienta en todo momento vinculado a la Universidad, apoyado e incentivado por ella, y se sienta también miembro de un grupo-referente, que vive su misma experiencia y se enfrenta de algún modo al mismo tipo de problemas con los que él debe tratar en su trabajo de estudio cotidiano.. 
El uso didáctico de las nuevas tecnologías, en el caso que nos ocupa del Campus Virtual como espacio de interacción y vida académica, ofrece algunas ventajas a la docencia: por una parte, la rapidez de la conexión a través del correo electrónico facilita la cantidad, la flexibilidad y el grado de individualización de los contactos entre profesor- estudiante, que reciben de manera inmediata, a cualquier hora del día en que abran su correo, en su buzón electrónico personal, el mensaje del emisor; por otra parte, el texto escrito del mensaje en el Campus Virtual se convierte en el vehículo de comunicación por excelencia, con las consecuencias que de ello se derivan, es decir, un canal imaginario y "virtual" de presencia del profesor, no por ello menos directo o interpelante, a menudo incluso más que la presencia real. El correo electrónico y el Campus Virtual como espacios tecnológicos tamizan, pues, la acción docente, dotando al sistema de enseñanza de una mayor flexibilidad, permanencia e inmediación, y proporcionando al estudiante una atención personalizada a través de la presencia "virtual" del profesor.

El docente actúa, en el marco que hemos definido, desde tres grandes modos de acción que pretenden motivar en el estudio, mantener el interés, así como facilitar el vínculo social, académico e institucional del estudiante con la universidad.

Algunas de las acciones básicas serían:

- Comunicarse con los estudiantes y hacer un seguimiento. Tomar iniciativas de comunicación, favorecer la continuidad en la relación personalizada; seguir el grado de progreso en los estudios, valorando éxitos y fracasos, ajustando el ritmo de trabajo a la disponibilidad del estudiante, así como también proponiendo nuevas formas de aprovechamiento y gestión del tiempo.

- Atender consultas, relativas a los estudios en aspectos académicos generales: elección de optativas, participación en encuentros, etc...; sobre informaciones relativas a la orientación profesional de su carrera; sobre cuestiones administrativas en relación a los estudios (número de convocatorias a exámenes, anulación y ampliación de matrículas, etc...) .

-Mantener contactos directos con los estudiantes, en presencia real -aprovechando los encuentros de cada semestre-; facilitando la identificación y la interacción del grupo de estudiantes entre sí, y sugerir datos o recomendaciones para la evaluación de su ritmo de trabajo.

-Gestionar el aula virtual, elaborar el plan docente y adecuarlo al ritmo y el estilo de aprendizaje del grupo y de cada estudiante.

Anna Pagés Santacana

apages@campus.uoc.es

Psicología y Ciencias de la Educación

Universitat Oberta de Catalunya (UOC)

-42771657612011 Content-Disposition: form-data; name="camino"

Revelec2/revelec14 